\title{
Polarization of Ellerman bombs and the arch structure of active region: some results of investigation
}

\author{
L.K. Kashapova \\ Institute of Solar-Terrestrial Physics RAS, P.O. Box 4026, Irkutsk, 664033, Russia \\ email: lkk@iszf.irk.ru
}

\begin{abstract}
The comparison of the polarization presence in Ellerman bombs or moustaches and the active region arch structure was carried out. In the most cases of the polarization, interpreted as impact polarization, were detected in that regions where the bright small loops were observed. On the contrary, when a significant polarization wasn't detected the scanning during observation was carried out nearby the large loops.
\end{abstract}

\section{Introduction}

Ellerman bombs or moustaches are enigmatic phenomena in the solar atmosphere. There are the several models suggesting explanation for so fast and at the same time so local energy release. The polarization observations take up the special place among the methods of the moustache investigation. For example, only they allow to extract moustaches which emission were excited by beams of energetic particles. The results of polarization observation as well as some results of comparison of birefringent filter observations with magnetic field maps enable to assume that different moustaches arise from different mechanisms. However the influence of a arch structure on the processes of Ellerman bomb formation and the results of polarimetric observations is still not enough studied. The aim of this work is analysis of relations between the polarimetric data and the arch structure of corresponding active regions.

\section{Observations}

The spectropolarimetric observations of Ellerman bombs were obtained in 1999-2000 on Large Solar Vacuum Telescope. The observational parameters and the methods of the polarization observations were described in Kashapova (2003) in detail. The EIT data (obtained by $\mathrm{SOHO}$ ) at the respective moments of observations were used for the arch structure investigation.

\section{Discussion}

As it was shown in Kashapova (2003), the spectropolarimetric observations in the hydrogen $\mathrm{H} \alpha$-line revealed the two groups of the moustaches. In the first group the linear polarization exceeded $3 \%$ and it was observed only in the line center. In the second group the polarization was insignificant. According to Zharkova \& Syniavskii (2000), the non-thermal mechanism is the most acceptable for the first group. In that case, the some features would be presented in the arch structures of active region (AR) which usually observed in UV-lines forming into the transition region. The former researchers either hadn't found such features (Payn 1993) or have been looking them in $1600 \AA$ 

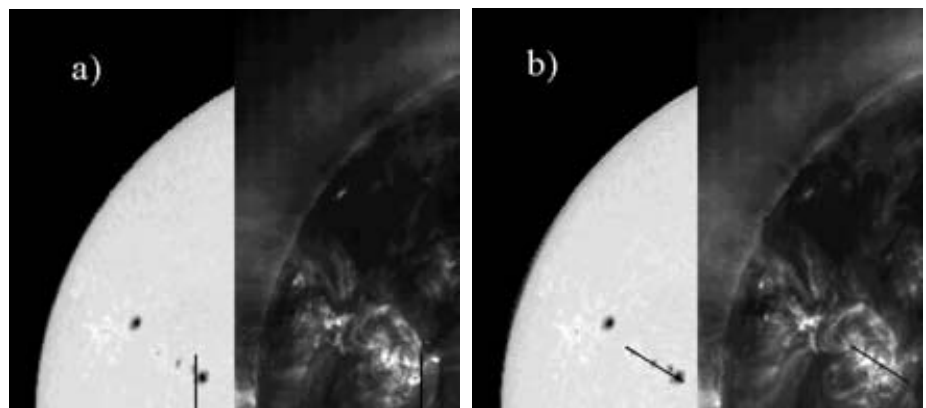

Figure 1. The image of AR 9114 on 06.08 .00 in whitelight (MDI, the right panel), and in $195 \AA$ line (EIT SOHO, the left panel). The direction of the slit during scanning of AR is shown on both panels. a)-UT $2^{h} 24^{m}$, the linear polarization in moustaches is less then $2 \%$. b)-UT $6^{h} 48^{m}$, the linear polarization in moustaches is $3-7 \%$.

continuum, corresponding to the temperature minimum region. (for example, Georgoulis et al. 2002). A comparison of $\mathrm{AR}$ arc structures by $195 \AA$ line images for the different groups of Ellerman bombs showed that in most cases of the polarization, interpreted as impact polarization, were detected in that regions where the bright small loops were observed (figure 1b). In those cases, when a significant polarization wasn't detected the scanning during observation was carried out nearby the large loops (figure 1a). The structure of active regions shown on figure $1 \mathrm{~b}$ is allied to AR considered in Schmieder et al. (2002) where the authors demonstrated a close relation of Ellerman bombs and magnetic reconnections.

In several cases, small bright loops were observed into AR but a significant polarization was not detected. It's worth to mention that both AR located within the range from 40 to 60 degrees from the solar disk center where the rate of moustaches with insignificant polarization is abnormally high. But for all that this ratio is in good agreement with the result presented in Rust \& Keil (1992). Such distribution is very difficult to explain only by the scattering peculiarities. Possibly, these moustaches also arose as a result of action of energetic particle beams but we could not observed the polarization because of a unaccounted quality of the impact polarization.

\section{Acknowledgements}

This work was supported by grant NSh-733.2003.2 of the State Support of Leading Scientific Schools of Russia Federation and the State Scientific Technical Program "Astronomy". Author acknowledges the SOHO Archive (http://sohowww.nascom.nasa.gov) for providing data for use in this publication.

\section{References}

Georgoulis, M.K., Rust, D.M., Bernasconi, P.N. \& Schmieder, B. 2002 Astrophysical Journal $\mathbf{5 7 5}, 506-528$.

Kashapova, L.K. 2003 Solar polarization 3 ASP Conference Series 307, 474-479.

Payne, T.E.W. 1993 Bulletin of the American Astronomical Society 25, 1395.

Rust, D.M.\& Keil S.L. 1992 Solar Phys. 140, 55-65.

Schmieder, B., Pariat, E., Aulanier, G., Georgoulis, M.K., Rust, D.M. \& Bernasconi P.N. 2002 Proc. 10th. European Solar Physics Meeting "Solar Variability: From Core to Outer Frontiers" SP-506, 911-914.

Zharkova, V.V. \& Syniavskii D.V. 2000 A $\& A$ 354, 714-724. 